International Journal of Electrical Engineering and Technology (IJEET)

Volume 11, Issue 4, June 2020, pp. 141-149, Article ID: IJEET_11_04_016

Available online at https://iaeme.com/Home/issue/IJEET? Volume $=11 \&$ Issue $=4$

ISSN Print: 0976-6545 and ISSN Online: 0976-6553

DOI: https://doi.org/10.34218/IJEET.11.4.2020.016

\title{
PERFORMANCE COMPARISON OF TECHNIQUES FOR INTERFERENCE COORDINATION IN HETNETS OF LTE-A
}

\author{
Koiloth SRS Jyothsna, Tummala Aravinda Babu, Joseph Michael Murray \\ Department of Electronics and Communication Engineering, CBIT- Chaitanya Bharathi \\ Institute of Technology, Hyderabad, Telangana, India.
}

\begin{abstract}
In mobile communication, the concept of heterogeneous networks has recently attracted significant importance as a way to optimize the performance of the network, especially for unequal user or traffic distribution situations. A heterogeneous network is poised of multiple radio access technologies, architectures, transmission solutions, and base stations of varying transmission power that can interoperate, thus creating a multilayer structure. Due to the different operating modes of the nodes, some of them work in open access mode but others work in closed access mode, and the unbalanced transmission power of the different base stations of the network, select the appropriate server station can be challenging for the users equipment. A wrong cell selection process can lead to the under-utilization of low power nodes; so that, range extension technique is proposed to allow more users to be attached to low power nodes. Management of interferences caused by the macro station to the low power nodes and vice versa is one of the biggest challenges in the deployment of heterogeneous networks. In this paper, the performance of convention techniques is evaluated and compared against the advanced techniques of LTE-A.
\end{abstract}

Key words: HetNet, LTE, intra frequency interference, interference coordination

Cite this Article: Koiloth SRS Jyothsna, Tummala Aravinda Babu, Joseph Michael Murray, Performance Comparison of Techniques for Interference Coordination in HetNets of LTE-A, International Journal of Electrical Engineering and Technology, 11(4), 2020, pp. 141-149.

https://iaeme.com/Home/issue/IJEET?Volume $=11 \&$ Issue $=4$

\section{INTRODUCTION}

In very recent years, mobile broadband traffic has grown exponentially, exceeding voice, thanks to the new generation of mobile terminals, such as smartphones, tablets and laptops, and to the new services and capabilities they offer. Mobile users have also increased and, with them, the number of connections. Furthermore, cellular operators have in general reported non-uniform traffic distributions in their networks, stating that for instance 50\% of the total traffic volume is carried on only $30 \%$ of the macrosites. The number of wireless subscribers 
have increased exponentially with time and telecommunication companies are perennially challenged to meet the customers' requirements for increased coverage and faster data transfer rates. The release 8/9 standard provides major benefits in High Speed Packet Access (HSPA) which includes better spectral efficiency, reduced latency on account of its Internet Protocol (IP) architecture, and greater throughputs.

Heterogeneous Networks (HetNet) are multi-mode, multi-layer and multi band structured and utilize cells of varying sizes.

The goal behind the design and implementation of HetNets involves incrementing the capacity of the established network, modifying spectrum use, lowering the capital and operating costs, and offering steady user-based experience of network architecture.

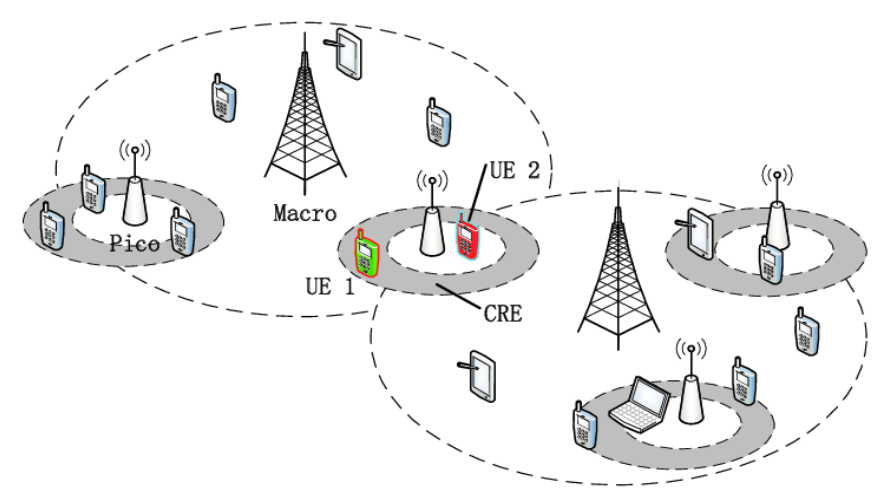

Figure 1 Typical HetNet Architecture in LTE-A

The traditional macro cells are still needed to provide general coverage, and connectivity particularly for those in outlying areas or in fast moving in vehicles where multiple handovers are not desirable. However, where high data rates are needed in buildings or urban areas, a variety of small cell technologies can be used. These may use one of a variety of backhaul technologies. However, the most important aspect is that, these need to appear as a single network to the user, providing the same enhanced performance level.

\subsection{Different Types of Cells and their Characteristics are as under}

a. Macro cells are the common cells sites supporting technologies like HSPA+ and LTE. The normal range may vary from a few hundred meters to a few kilometers. Output power is of the order of tens of watts.

b. Microcells typically cover smaller areas maybe up to a kilometer. They usually transmit within a range of mill watts to a few watts. Microcells are deployed for providing temporary cellular coverage and capacity to places like sports stadiums, convention centers etc. Sometimes, microcells may use distributed antenna systems (DAS) to improve bandwidth and reliability.

c. Pico cells offer capacities and coverage areas, supporting up to 100 users over a range of less than 250 yards. Pico cells are frequently deployed indoors to improve poor wireless and cellular coverage within a building, such as an office floor or retail space.

d. Femtocells are typically user-installed to improve coverage area within a small vicinity, such as home office or a dead zone within a building. Femtocells can be obtained through the service provider or purchased from a reseller. Unlike pico cells and microcells, femtocells are designed to support only a handful of users and is only capable of handling a few simultaneous calls. They are sold by the operator but self-installed by the customer. 
However, these random small cell deployments cause severe problems and results interference in the network. Therefore, the ultimate is the total system performance degradation and this interference becomes a key challenge in HetNets.

\section{IMPLEMENTATION OF HETNET IN LTE-A}

In heterogeneous networks the cells of different sizes are referred to as macro, micro, pico and femto-cells; listed in order of decreasing base station power. In LTE networks, the actual cell size depends not only on the eNodeB power but also on antenna position, as well as the location environment; e.g. rural or city, indoor or outdoor etc. Different nodes, for small cells, used in LTE/LTE-A HetNets are listed below:

1.Home eNodeB (HeNB), a 3GPP term for femto-cell in LTE, was introduced in LTE Release 9 (R9). It is a low power eNodeB which is mainly used to provide indoor coverage, for Closed Subscriber Groups (CSG), for example, in office premises. HeNBs are privately owned and deployed without coordination with the macro-network. If the frequency used in HeNB is the same as the frequency used in the macro-cells, and the HeNB is only used for CSG, then there is a risk of interference between the HeNB and the surrounding network.

2. Relay Node (RN) is another type of low-power base station added to the LTE R10 specifications. In LTE-Advanced, the possibility for efficient heterogeneous network planning is increased by the introduction of Relay Nodes (RNs). The Relay Nodes are low power base stations that will provide enhanced coverage and capacity at cell edges, and hot-spot areas and it can also be used to connect to remote areas without fibre connection.

3. RRHs (Remote Radio Head) connected to an eNB via fibre can also be used to provide small cell coverage. It is an alternative solution to a BTS housed in a shelter at the bottom of the tower. It is a distributed base station, in which the majority of the base station equipment is no longer located in the shelter, but in an enclosure at the top of the tower near the antennae. This separate but integrated radio frequency (RF) unit is called a remote radio unit or remote radio head. It is compact in size. RRH is generally used to extend the coverage of a base station sub-system in the remote rural areas.

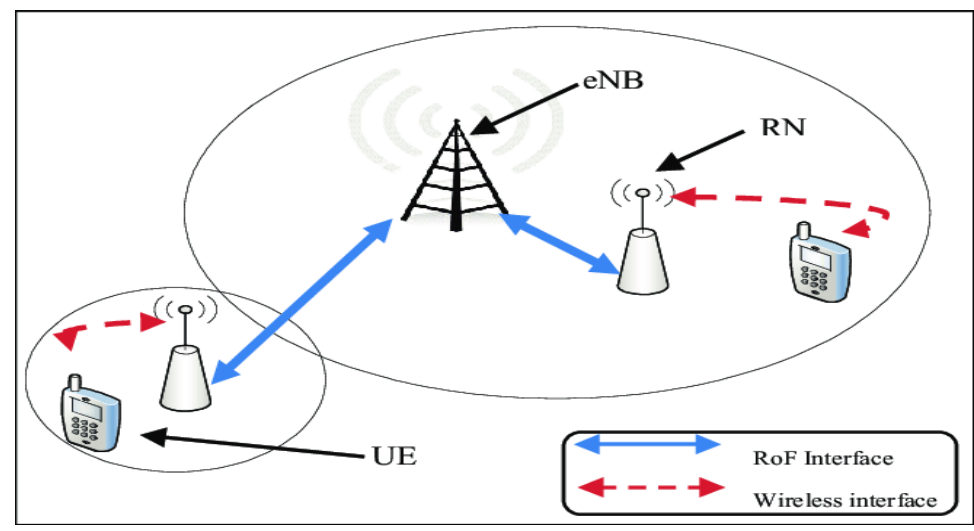

Figure 2:HetNet with Relay Node

Introducing a mix of cell sizes and generating a heterogeneous network adds to the complexity of network planning. In a network with a frequency reuse of one, the UE normally camps on the cell with the strongest received DL signal (SSDL), hence the border between two cells is located at the point where SSDL is the same in both cells. In homogeneous networks, this also typically coincides with the point of equal path loss for the UL (PLUL) in both cells. In a heterogeneous network, with high-power nodes in the large cells and lowpower nodes in the 7 small cells, the point of equal SSDL will not necessarily be the same as that of equal PLUL. 
A major issue in heterogeneous network planning is to ensure that the small cells actually serve enough users. One way to do that is to increase the area served by the small cell, which can be done through the use of a positive cell selection offset to the SSDL of the small cell. This is called Cell Range Extension (CRE).

\section{TECHNIQUES FOR INTERFERENCE COORDINATION IN HETNETS}

In order to evade interferences, HeNodeBs synchronization is veritably important. The cochannel deployment in macro-eNodeB and HeNodeBs could increase the capacity of the network manifold through high spatial frequency reuse. However, co-channel deployment in macroeNodeB and HeNodeBs results interference in the network which becomes a key challenge in HetNet.

Inter-cell Interference Coordination (ICIC): ICIC was introduced in R8. The eNBs can communicate using ICIC via the X2 interface to mitigate inter-cell interference for UEs at the cell edge. The X2AP message used for this is called "Load Information".Through the "Load Information" message an eNB can inform neighbouring eNBs about: UL interference level per Physical Resource Block (PRB); UL PRBs that are allocated to cell edge UEs, and hence are sensitive to UL interference; and if DL Tx power is higher or lower than a set threshold value. The eNBs receiving these messages can use the received information to optimize scheduling for UEs at cell edges.

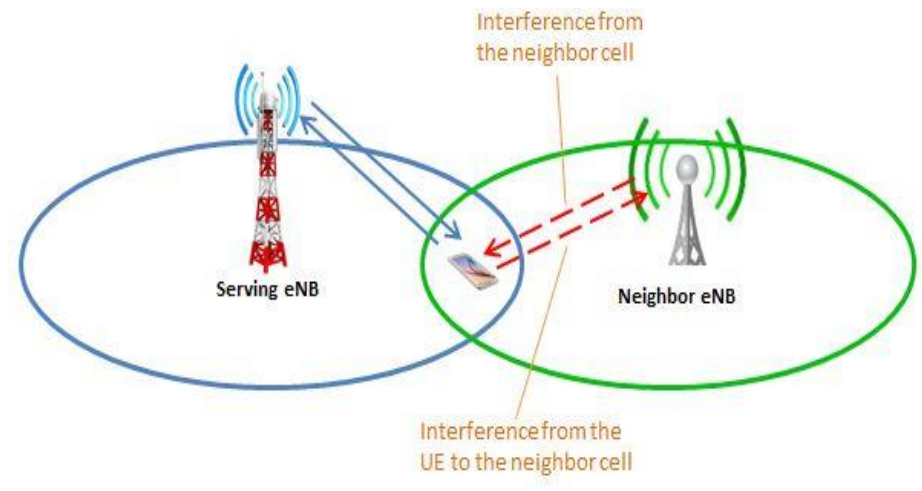

Figure 3 Inter-cell Interference Coordination in LTE-A

Enhanced Inter-Cell Interference Coordination: It is the primary interference management and mitigation procedure adopted in the LTE-A network. It is typically used in a heterogeneous network where both macro and other small cells transmit and receive data at the same time. The weaker signal from the smaller cell can be easily overpowered by the stronger signal from the larger cell. Advanced interference mitigation schemes have been used in LTE networks, but with the increasingly high density of wireless network cells, more sophisticated schemes like eICIC are required.

eICIC introduces concept of "Almost blank subframe" (ABS). ABS subframes do not send any traffic channels and are mostly control channel frames with very low power. If macro cell configure ABS subframes then UEs connected to pico/femto cells can send their data during such ABS frames and avoid interference from macro cell.

eICIC mitigates interference on traffic and control channels. It uses power, frequency and also time domain to mitigate intra frequency interference in heterogeneous networks. 


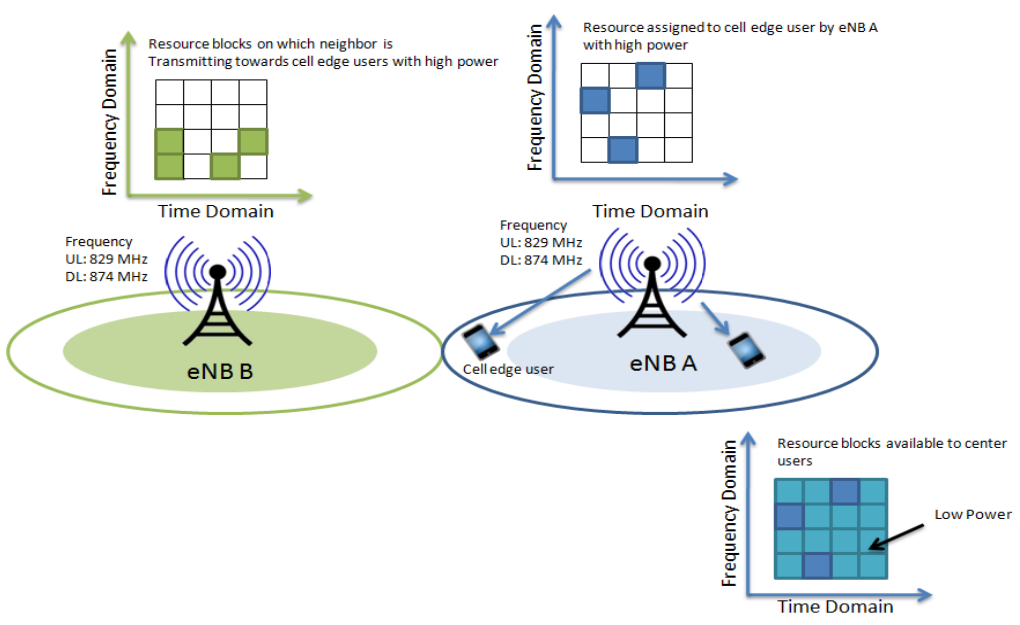

Figure 4 ICIC and Enhance ICIC in LTE-A

Coordinated Multipoint (CoMP) Transmission: This was formalized in 3GPP Release 11. CoMP is another key characteristic of a LTE Advanced network. In a Coordinated multipoint transmission and reception scenario, multiple eNodeBs work with each other dynamically to avoid interference with other transmission signals. This leads to a better utilization of system resources and an enhancement of both network coverage and quality for cell edge users.
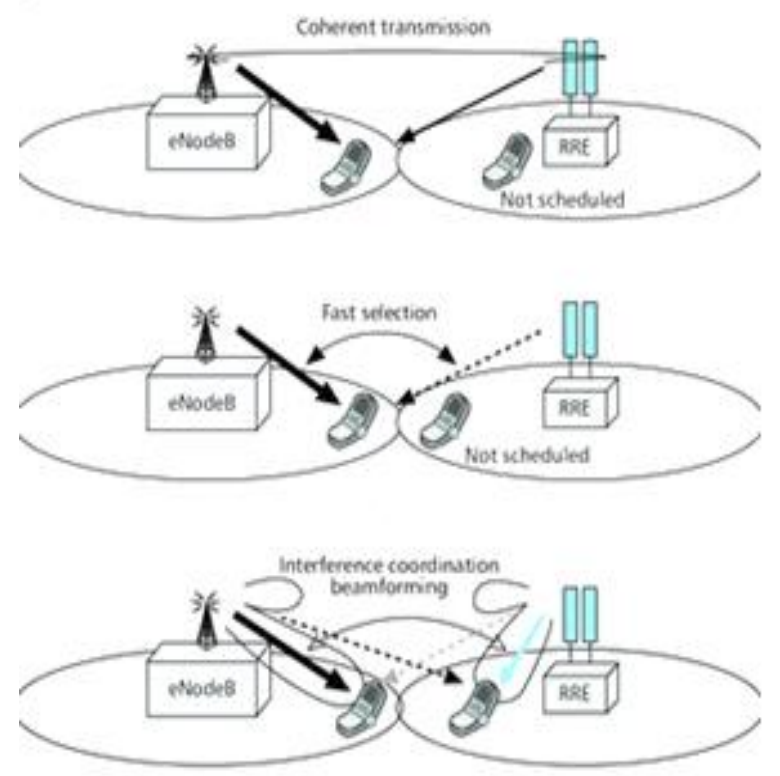

Figure 5 Various scenarios in CoMP transmission

Standardized network-based inter-cell interference coordination (ICIC) schemes for LTE range from basic coordination to further enhanced ICIC, and more elaborate coordinated multi-point (CoMP) communication techniques.

\section{COMPARATIVE ANALYSIS OF THE BASIC SCHEMES AND INTERFERENCE COORDINATION IN 5G RADIO}

The solutions for LTE were mainly designed to offer spectral efficiency benefits for data channel transmissions by applying various forms of intercell coordinated muting (or power adjustments) or interference randomization, while offering only limited benefits for control channel performance. 
The following are the schemes used for interference mitigation in 5G communication:

Uplink Inter-cell Pilot coordination: In UL, inter-cell pilot interference arises when the assigned uplink pilot sequences across multiple cells, which are non-orthogonal, are scheduled on the same time-frequency resources. The received pilots from a target user suffer from pilot interference coming from neighboring cells, resulting in poor channel estimation. This problem is especially severe for cell-edge users, as the power of the interfering pilots is comparable to that of desired pilots. It leads to errors in uplink coherent demodulation, and it is very detrimental in uplink multi-user MIMO scenarios that heavily rely on accurate channel knowledge to perform receive filtering. Furthermore, the BSs treat pilot interference as noise, which is suboptimal at high uplink signal-to-noise ratios (SNRs) occurring in, for example, small cell scenarios. In such scenarios, a better approach would be the suppression of pilot interference at the BS to recover the desired pilots with as little interference as possible.

Downlink Inter-cell Interference coordination: In the DL, the trend is toward more dynamic ICIC solutions, as already agreed in $3 \mathrm{GPP}$ for $5 \mathrm{G}$, as well as addressing various network deployments (small cell and macro scenarios), key $5 \mathrm{G}$ technologies (dense small cell networks and MU-MIMO), and KPI requirements (spectral efficiency, energy, and reliability).

Downlink Inter-cell coordinated small cell DTX: Cell discontinuous transmission is an energy saving technology that adapts the cell activity to its instantaneous load. Within each frame, the cell DTX will instantaneously activate (deactivate) the cell components and the associated functionalities when the user data is present (absent) in the cell queue. Radio resource management (RRM) and lower-layer functions are implemented locally at the transmission points; thus, the controller and the small cells do not need to continuously exchange messages through the backhaul. On the contrary, a fully distributed architecture requires coordination across nearby small cells, which in turn increases the small cell (and the backhaul) energy consumption. In the same way, implementing centralized scheduling or coordinated beamforming schemes at the network controller 1) increases network complexity; 2) needs regular transmission of channel quality indicators (CQIs) over the backhaul link; and 3 ) is affected by the backhaul latency and capacity constraints. In any case, the proposed solution with reinforcement learning manages the small cell activity to limit network energy consumption without reducing the system quality of service (QoS).

Downlink Inter-cell Rank Adaptation: In a MIMO setting, the downlink serving rank (or number of transmission streams) plays a major role in the interference suppression levels of interference rejection combining (IRC) receivers. This is because significant interference suppression is only possible when the number of desired data streams and dominant interference streams are collectively less than the receiver dimension, i.e., the number of receive antennas. Traditionally, rank selection at each user is essentially performed in a selfish manner independently per link, without taking into account the interference caused by such selections. For 5G NR, an inter-cell rank coordination mechanism can improve the network and the cell edge user throughput by coordinating the generated inter-cell interference from the aggressor cell.

The proposed coordination mechanism involves the following steps, as:

1) The UEs report the DIR and the CQI to the serving BS. The serving BS determines whether the DIR is above a certain pre-specified threshold, and rank coordination is only invoked for those UEs with a strong DI. The UEs selected for rank coordination are then grouped according to the DI, to avoid conflicting coordination requests from the same BS.

2) The serving BS decides what will be the maximum transmission rank for each of the UEs in each group, along with the interference rank it would like to have. The signal to interference and noise (SINR) ratio is used for the decision. The ranks are chosen based on the 
estimated post IRC SINR.1 The proposed rank coordination mechanism is not bound to any specific rank adaptation algorithm, though interference-aware rank adaptation algorithms such as those presented are best suited for such applications.

3) The serving BS sends the desired rank message to the respective interfering BS. The desired rank message is indicated as the maximum allowable transmission rank for a given set of PRBs. These messages can be per single PRB, or several PRBs can be grouped into a single desired rank and priority level. The granularity provides a tradeoff among performance, overhead and complexity.

4) The serving BS updates its transmission parameters according to the feedback message from the interfering BS. Such updates can include re-scheduling the users, re-adjusting the transmission parameters, or re-adapting the transmission rank with respect to the feedback message. The 5G TTI is expected to be shorter than the current $1 \mathrm{~ms}$ of LTE. The rank coordination could occur over a longer time basis (in the range of 5-10 ms), therefore suitable for heavy payload traffic spanning over multiple TTIs. For random intermittent traffic with small payload, the interference rank can be pre-coordinated to cater to such bursty but critical payloads.

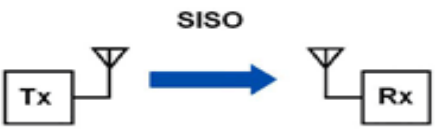

(a)

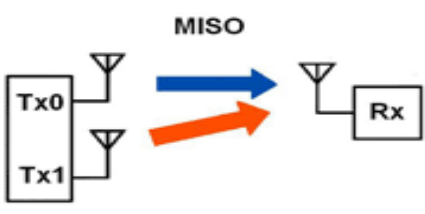

(c)
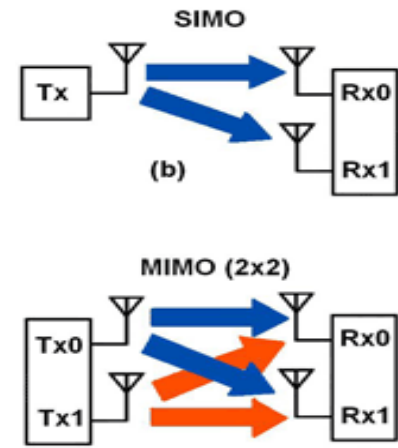

(d)

Figure 6 Types of inputs and outputs in LTE MIMO

\section{RESULTS AND DISCUSSIONS}

In this paper, the performance comparison of the basic interference coordination techniques with those novel techniques in 5G of LTE-A is presented by considering throughput and SNR as performance measures.

The following figure shows the throughput analysis of Single Input Multi Output (SIMO). It is found that the throughput of 4 receiving antennas is better compared with 2 receiving antennas.

The preliminary analysis shown below is only with Single Input and Multi Output (SIMO) with only two configurations. 


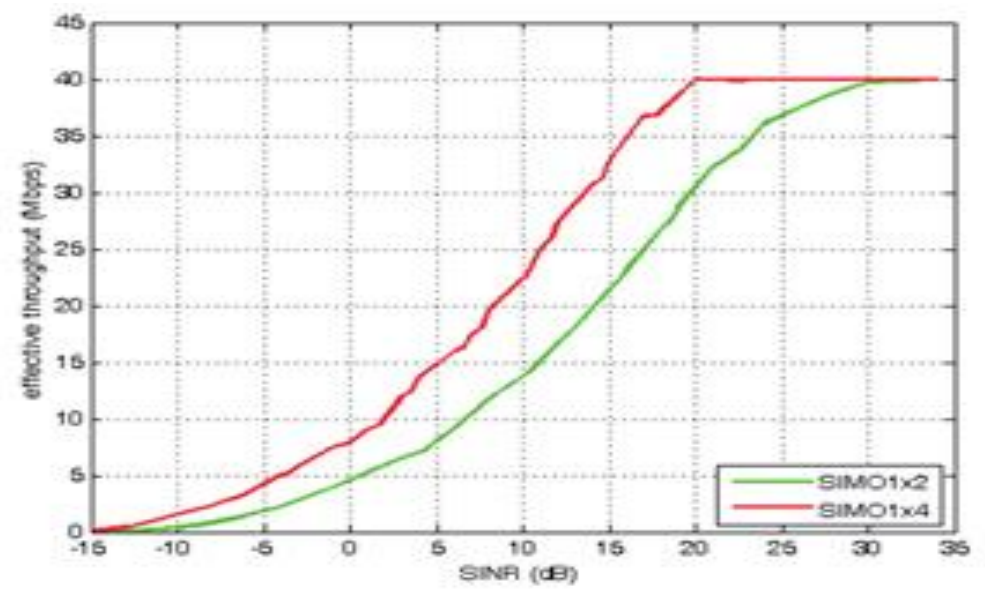

Figure 7 Throughput Vs SNR in SIMO Scenario

However, LTE-A uses MIMO systems for better performance. The following table shows the throughput analysis of SIMO and 2X2 MIMO:

Table 1 Throughput analysis between SISO and 2X2 MIMO for various system bandwidths:

\begin{tabular}{|c|c|c|}
\hline $\begin{array}{c}\text { Transmission Mode/ } \\
\text { System Bandwidth } \\
\text { (MHz) }\end{array}$ & $\begin{array}{c}\text { SISO } \\
\text { Downlink Peak } \\
\text { throughput (Mbps) }\end{array}$ & $\begin{array}{c}\text { 2X2 MIMO } \\
\text { Downlink Peak } \\
\text { throughput (Mbps) }\end{array}$ \\
\hline 1.4 & 4.4 & 8.8 \\
\hline 3 & 11.1 & 22.1 \\
\hline 5 & 18.3 & 36.7 \\
\hline 10 & 36.7 & 75 \\
\hline 15 & 55.1 & 110 \\
\hline 20 & 75 & 150 \\
\hline
\end{tabular}

From the table 1 , it is very clear that the MIMO techniques used in LTE-A has given almost double the throughput for the same frequency of operation. Hence, Uplink Inter-cell Pilot coordination and Downlink Inter-cell Interference coordination used in LTE-A has given twice the performance as those of conventional schemes. This is because of employment MIMO systems in LTE-A compared to LTE.

\section{CONCLUSIONS}

In LTE-A, HetNets plays a vital role. HetNet architecture allows capacity expansion to be based on actual demand in data traffic. By deploying small cells and HetNet networks, operators can address both short term and long-term challenges to enhance the network capacity and quality. Optimizing current macro cell and future heterogeneous networks requires a multi- dimensional approach. Hence, in this paper several conventional techniques are discussed. In comparison to those conventional techniques, the techniques used in advanced LTE systems are evaluated. The performance of both is analyzed in terms of throughput. It is evident from the results, that LTE-A with MIMO configuration has given better performance. The performance can be further increased by increasing the number of transmitting and receiving antennas at the expense of system complexity. 


\section{REFERENCES}

[1] B. Soret et al., (2015) "Interference Coordination for Dense Wireless Networks," IEEE Commun. Mag., vol. 53, no. 1, Jan. pp. 102-09.

[2] G. Boudreau et al., (2009) "Interference Coordination and Cancellation for 4G Networks," IEEE Commun. Mag., vol. 47, no. 4, Apr. pp. 74-81.

[3] K. I. Pedersen et al., (2013) "Enhanced Inter-Cell Interference Coordination in CoChannel Multi-Layer LTE-Advanced Networks," IEEE Wireless Commun, vol. 20, no. 3, June, pp. 120-27.

[4] 3GPP Technical Report 38.801, (2016) "Study on New Radio (NR) Access Technology; Radio Access Architecture and Interfaces," Dec.

[5] 3GPP Technical Report 38.802, (2017) "Study on New Radio (NR) Access Technology; Physical Layer Aspects," Jan.

[6] 3GPP Technical Report 38.913, (2016) "Study on Scenarios and Requirements for Next Generation Access Technologies,” March.

[7] W. Nam et al., (2014) "Advanced Interference Management for 5G Cellular Networks," IEEE Commun. Mag., vol. 52, no. 5, May, pp. 52-60.

[8] N. H. Mahmood, K. I. Pedersen, and P. Mogensen, (2017) "Interference Aware Inter-Cell Rank Coordination for 5G Systems," IEEE Access, DOI: 10.1109/ACCESS.2017.2672799.

[9] B. Soret and K. I. Pedersen, (2017) "Power Boost and Cell Muting for High Reliability and Low Latency in 5G," Proc. IEEE Vehicular Technology Conf. (VTC), Spring.

[10] J. W. Kang et al., (2011) "Optimal Pilot Sequence Design for MultiCell MIMO-OFDM Systems," IEEE Trans. Wireless Commun, vol. 10, no. 10, Oct. pp. 3354-67.

[11] V. Fernandez-Lopez et al., (2016) "Improving Dense Network Performance through Centralized Scheduling and Interference Coordination,” IEEE Trans. Veh. Technol., DOI: 10.1109/ TVT.2016.2609239. 